\title{
Behavioural Impairment and Frontotemporal Dementia in Oculopharyngeal Muscular Dystrophy
}

\author{
Maurits Tankink ${ }^{\mathrm{a}, *, 1}$, Corinne G.C. Horlings $\mathrm{s}^{\mathrm{b}, \mathrm{c}, 1}$, Nicol Voermans ${ }^{\mathrm{b}}$, Barbara van der Sluijs ${ }^{\mathrm{d}}$, \\ Roy P.C. Kessels ${ }^{\mathrm{e}, \mathrm{g}}$, Baziel van Engelen ${ }^{\mathrm{b}}$ and Joost Raaphorst ${ }^{\mathrm{f}}$ \\ ${ }^{a}$ Department of Neurology, Radboud University Medical Center, EX Nijmegen, the Netherlands \\ ${ }^{\mathrm{b}}$ Department of Neurology, Donders Institute for Brain, Cognition and Behaviour, Radboud University Medical \\ Center, EX Nijmegen, the Netherlands \\ ${ }^{\mathrm{c}}$ Department of Neurology, Medical University Innsbruck, Innsbruck, Austria \\ ${ }^{\mathrm{d}}$ Department of Neurology, Gelre Hospital Zutphen, Zutphen, The Netherlands \\ ${ }^{\mathrm{e}}$ Donders Institute for Brain, Cognition and Behaviour, Radboud University. HR Nijmegen, The Netherlands \\ ${ }^{\mathrm{f}}$ Amsterdam UMC, University of Amsterdam, Department of Neurology, Amsterdam Neuroscience, Amsterdam, \\ Netherlands \\ ${ }^{\mathrm{g}}$ Department of Medical Psychology, Radboud University Medical Center, EX Nijmegen, the Netherlands
}

Pre-press 28 July 2021

\begin{abstract}
Some patients with Oculopharyngeal Muscular Dystrophy (OPMD) develop frontotemporal dementia (FTD). The prevalence and clinical correlates of behavioural impairment, including FTD, is unknown in OPMD.

24 OPMD patients and their proxies completed a questionnaire concerning behavioural impairment (ALS-FTD-Q). We examined proportions with mild or severe behavioural changes, according to validated cut-off proxy scores. We examined correlations with the Hospital Anxiety and Depression Scale (HADS), the Short Form Health Survey (SF-36), motor symptoms, genotype and disease duration.

In this small patient sample, behavioural impairment was present in $29 \%$ of OPMD patients; in $17 \%$ the severity of symptoms was compatible with bvFTD. Correlations were small to medium.
\end{abstract}

Keywords: Oculopharyngeal muscular dystrophy, frontotemporal dementia, behavioural impairment, neuromuscular disorders, neuropsychiatry

\section{INTRODUCTION}

Oculopharyngeal muscular dystrophy (OPMD) is a hereditary muscular dystrophy caused by

\footnotetext{
${ }^{1}$ Both authors contributed equally.

*Correspondence to: Maurits Tankink, Department of Neurology, Radboud University Medical Center. Internal code 943, Reinier Postlaan 4, 6525 EX Nijmegen, the Netherlands. Tel.: +31 06 40676924; E-mail: maurits.tankink@gmail.com.
}

a GCN-repeat expansion in the Poly-AdenylateBinding-Protein-Nuclear 1 gene (PABPN1) [1]. Typically, OPMD patients show ptosis, dysphagia and limb-girdle weakness. Brain involvement, characterized by frontotemporal lobe degeneration [2], may occur in a proportion of patients with OPMD. Case reports of patients with OPMD described behavioural and cognitive changes, including executive dysfunction, apathy and visual hallucinations 
[3], which may hamper daily functioning and can be classified as frontotemporal dementia (FTD) in some cases [4]. A neuropsychological study in 11 patients with OPMD showed mild executive dysfunction and a correlation between MMSE scores and GCN-repeat length [3]. In another study, psychosis was reported in a subset of seven out of 10 patients with a homozygous GCN-[13/13] repeat [2].

Based on these previous reports, the extent and severity of behavioural changes in OPMD, including behavioural variant FTD (bvFTD; a clinical syndrome characterized by progressive deterioration of behaviour and personality), could be substantial, clinically relevant and related to GCN-repeat length, but this has not been systematically evaluated.

We examined the prevalence and severity, as well as the clinical and genetic correlates of behavioural changes including bvFTD, in a cohort of patients with genetically defined OPMD.

\section{MATERIALS AND METHODS}

\section{Participants}

Patients with genetically confirmed OPMD were recruited from the national neuromuscular database (CRAMP) in the Netherlands [5]. Proxies (spouses or other family members who were able to judge the patient's behaviour) were asked to participate. Exclusion criteria were: a) inability to speak or write Dutch fluently, b) current or past other neurological disorders with central nervous system involvement, and c) unavailability of a proxy.

\section{Standard protocol approvals, registrations, and patient consents}

The local ethics committee of the participating hospital approved the study. Written informed consent was obtained from all participants.

\section{Questionnaires}

\section{ALS-FTD- $Q$}

The ALS-FTD-Q is an observer reported scale filled out by the proxy of a patient (www.alsftdq.nl). The ALS-FTD-Q is a validated instrument for detecting behavioural changes related to bvFTD in patients with ALS, and is described in detail elsewhere [6]. The items of the ALS-FTD-Q are phrased to minimalize impact of motor and speech dys- function. The ALS-FTD-Q aims to assess various domains of behaviour (e.g. disinhibition, apathy, executive dysfunction, emotional expression, social behaviour/empathy). For this study, we used previously validated cut off scores of $<22$ (no behavioural impairment), $\geq 22$ and $<29$ (mild behavioural change) and $\geq 29$ (severe behavioural change, compatible with a diagnosis bv-FTD). By means of an exploratory part of the study, the patients filled in the ALSFTD-Q as well, in order to obtain a measure of the patients' perception of their own behavioural impairments.

To examine associations of behavioural impairment (as scored by proxies) with symptoms of anxiety/depression, quality of life, and motor and speech impairment, we used the following questionnaires which were filled out by the patients:

1) Hospital Anxiety and Depression Scale (HADS). Scores of 0-7 are normal, scores of 8-10 are borderline abnormal, and scores of 11 or higher are indicative of clinically relevant affective symptoms [7].

2) SF-36. This generic Quality of life questionnaire consists of eight subscales. Scores are presented per subscale and have a maximum score of 100. A higher score corresponds with better quality of life (QoL) [8].

3) Dutch dysarthria investigation for adults; a validated 7-item questionnaire on dysarthria symptoms, with a 5-point scale (scores 0-4) and a maximum score of 28 . A higher score corresponds with more severe dysarthria [9].

4) Swallowing questionnaire; a 6-item questionnaire with 4/5/6-point scales (scores 0-5) and a maximum score of 24 . A higher score corresponds with more severe problems with swallowing [10].

\section{Procedure}

Following written informed consent, participants received an invitation by e-mail using Castor, a digital facility enabling participants to fill in the questionnaires online at home [11]. Participants without access to the internet received the questionnaires by postal mail.

The patients completed all the questionnaires and the proxies only completed the ALS-FTD-Q.

GCN repeat length of the PABPNI gene was collected from clinical files. 


\section{Statistical analysis}

We calculated the number of patients with abnormal proxy scores on the ALS-FTD-Q using the aforementioned cut-off values. To examine associations between behavioural changes and affective symptoms, quality of life, motor symptoms, genotype and disease duration, we calculated Spearman correlation coefficients $(\rho)$ of ALS-FTD-Q scores with scores of HADS, SF-36, Dutch dysarthria investigation for adults and the Swallowing questionnaire, as well as GCN repeat length and disease duration. Effect sizes were interpreted according to Cohen ( $\rho=0.1$ small, $\rho=0.3$ medium and $\rho=0.5$ large) [12].

In an exploratory fashion, differences between the ALS-FTD-Q scores of the proxy and the patient were examined with a Mann-Whitney U test, as a measure of the patients' insight into behavioural impairment. Analyses were performed in IBM SPSS Statistics 25.

\section{RESULTS}

We invited 76 patients (after a first screening of the exclusion criteria), 61 of whom responded, and 25 participated. One patient dropped out due to unexpected health problems. Demographics and results of the remaining 24 patients are shown in Table 1. The participants did not differ significantly from the nonparticipants in terms of $\operatorname{sex}(p=0.915)$ or age $(p=0.204)$.

Based on the ALS-FTD-Q scores as filled in by the proxies, seven out of 24 (29\%) patients showed behavioural changes: three patients $(12 \%)$ had mild, and four patients (17\%) had severe behavioural changes compatible with bvFTD. Based on the patients' scores of the ALS-FTD-Q, three out of 24 (13\%) patients showed mild behavioural changes. The ALS-FTD-Q median score of the patients (perception of their own behaviour), as compared to the proxy median score, was 10 (range 0-25) and 11 (range 0-58), respectively $(\mathrm{U}=255.5, \mathrm{z}=-0.671$, $p=0.502)$.

Correlations between the ALS-FTD-Q of the proxy and the HADS $(0.22, p=0.293)$, SF-36 subscales (ranging from -0.39 to $-0.07, p$-values ranging from 0.070 to 0.737 ; see Table 1 for all subscale correlations), Dutch dysarthria investigation for adults $(0.19, p=0.370)$, Swallowing questionnaire (0.12, $p=0.585), \mathrm{GCN}$-repeat $(0.31, p=0.141)$ and age $(0.21, p=0.334)$, age at onset $(0.18, p=0.429)$ and disease duration $(0.12, p=0.574)$ were small to medium. Three out of 24 patients (13\%) scored 11 or higher on the HADS, indicating clinically relevant affective symptoms; of whom two patients had clinically relevant depression scores. Out of these three patients, two also showed behavioural changes (one mild, one severe).

\section{DISCUSSION}

This study assessed behavioural impairment and bvFTD in a rare neuromuscular disorder caused by GCN-repeat expansion. The main finding in our sample of 24 patients with OPMD is that in $29 \%$, behavioural changes were observed. In $17 \%$, the severity of the behavioural changes was in the range of bvFTD.

The cited point prevalence of FTD (bvFTD and language variants taken together) of 15-22/100,000 from a 2013 review is considerably higher than the most detailed investigation in a Dutch region which showed a prevalence of 2.7/100.000 [13, 14]. The reasons for this discrepancy are beyond the scope of this discussion. When we presume a prevalence of $10 / 100.000$ in the Netherlands; the a-priori chance of one patient with (bvFTD) in a random sample of 24 people would be 0.0024 .

Despite the relatively low number of patients and the high rate of nonparticipating patients, these results further substantiate previous case reports suggesting brain involvement in OPMD [15, 16]. Indeed, frontotemporal atrophy was reported on MRI in single cases of OPMD [3]. Our findings further confirm that in neuromuscular diseases with repeat expansions (C9ORF72-related amyotrophic lateral sclerosis, myotonic dystrophy), brain involvement is a relatively frequent finding $[17,18]$. In ALS, the presence of frontotemporal dysfunction is an independent negative predictor of survival, and is related to restricted use of supportive therapies (e.g. feeding tube) [19]. A similar effect of FTD symptoms in OPMD on survival is likely, but should be subject of future research.

Mice and post-mortem human brain studies have also provided evidence for brain involvement in OPMD. In transgenic mice expressing the extended form of human PABPN1, ubiquitin-PABPN1positive intranuclear aggregates were found in neuronal cells [18]. Fluorescence immunohistochemistry using the same antibodies against PABPN1 on postmortem brain tissue of an OPMD patient showed intra-nuclear inclusions containing PABPN1 in cerebellar neurons (other brain regions were not studied) 
Table 1

Demographic, clinical, genetic and behavioural data of OPMD patients

\begin{tabular}{|c|c|c|c|c|c|c|c|c|c|c|c|}
\hline Case & Sex & Age, $y$ & $\begin{array}{c}\text { Age at } \\
\text { onset, y }\end{array}$ & $\begin{array}{c}\text { Disease } \\
\text { duration, y }\end{array}$ & $\begin{array}{c}\text { GCN expansion } \\
\text { a }\end{array}$ & $\begin{array}{l}\text { ALS-FTD-Q } \\
\text { score (proxy)* }\end{array}$ & $\begin{array}{c}\text { ALS-FTD-Q } \\
\text { score (patient)* }\end{array}$ & $\begin{array}{c}\text { HADS } \\
\text { score }\end{array}$ & $\begin{array}{l}\text { SF-36; PF } \\
\text { PF score }\end{array}$ & $\begin{array}{c}\text { SF-36; } \\
\text { RP score }\end{array}$ & $\begin{array}{c}\text { SF-36; } \\
\text { BP score }\end{array}$ \\
\hline 1 & $\mathrm{~F}$ & 56 & 50 & 6 & 10/16 GCN & 5 & $\underline{22}$ & $11 / 5$ & 65 & 25 & 31 \\
\hline 2 & $\mathrm{~F}$ & 44 & - & 0 & $10 / 13 \mathrm{GCN}$ & 0 & 5 & $1 / 0$ & 100 & 100 & 100 \\
\hline 3 & M & 71 & 54 & 17 & $10 / 16 \mathrm{GCN}$ & $\underline{24}$ & 11 & $0 / 3$ & 25 & 0 & 100 \\
\hline 4 & M & 57 & 35 & 22 & $12 / 16 \mathrm{GCN}$ & 58 & $\underline{23}$ & $1 / 0$ & - & - & - \\
\hline 5 & $\mathrm{~F}$ & 71 & 48 & 23 & 10/16 GCN & 11 & 9 & $3 / 4$ & 15 & 50 & 84 \\
\hline 6 & $\mathrm{~F}$ & 61 & 58 & 3 & $10 / 12 \mathrm{GCN}$ & 30 & 19 & $9 / 7$ & 80 & 25 & 62 \\
\hline 7 & $\mathrm{~F}$ & 66 & 46 & 20 & 10/14 GCN & 13 & 2 & $4 / 3$ & 30 & 100 & 51 \\
\hline 8 & $\mathrm{~F}$ & 65 & 36 & 29 & 10/16 GCN & 8 & 10 & $5 / 3$ & 25 & 25 & 74 \\
\hline 9 & $\mathrm{~F}$ & 58 & 48 & 10 & $10 / 12 \mathrm{GCN}$ & 1 & 1 & $0 / 0$ & 95 & 100 & 84 \\
\hline 10 & $\mathrm{~F}$ & 53 & 49 & 4 & $10 / 16 \mathrm{GCN}$ & 13 & 8 & $0 / 1$ & 100 & 100 & 100 \\
\hline 11 & $\mathrm{~F}$ & 57 & 50 & 7 & 10/16 GCN & 9 & 19 & $3 / 6$ & 35 & 0 & 41 \\
\hline 12 & $\mathrm{~F}$ & 57 & 53 & 4 & $10 / 16 \mathrm{GCN}$ & 2 & 5 & $4 / 2$ & 95 & 100 & 100 \\
\hline 13 & $\mathrm{~F}$ & 58 & 50 & 8 & $10 / 16 \mathrm{GCN}$ & $\underline{22}$ & 9 & $3 / 4$ & 75 & 100 & 74 \\
\hline 14 & M & 61 & 50 & 11 & 10/16 GCN & 31 & $\underline{25}$ & $3 / 13$ & 30 & 0 & 41 \\
\hline 15 & $\mathrm{~F}$ & 55 & 44 & 11 & $10 / 16 \mathrm{GCN}$ & 10 & $\overline{3}$ & $0 / 3$ & 60 & 100 & 100 \\
\hline 16 & M & 65 & 55 & 10 & $10 / 15 \mathrm{GCN}$ & 39 & 17 & $5 / 4$ & 25 & 25 & 41 \\
\hline 17 & M & 54 & 40 & 14 & $10 / 16 \mathrm{GCN}$ & $\underline{22}$ & 19 & $6 / 13$ & 30 & 25 & 32 \\
\hline 18 & $\mathrm{~F}$ & 58 & - & 0 & $10 / 16 \mathrm{GCN}$ & $\overline{15}$ & 0 & $1 / 1$ & 80 & 100 & 100 \\
\hline 19 & M & 66 & 60 & 6 & $10 / 14 \mathrm{GCN}$ & 11 & 14 & $2 / 2$ & 45 & 100 & 62 \\
\hline 20 & M & 71 & 47 & 24 & $10 / 14 \mathrm{GCN}$ & 12 & 11 & $7 / 8$ & 35 & 25 & 62 \\
\hline 21 & M & 56 & 45 & 11 & $10 / 16 \mathrm{GCN}$ & 3 & 10 & $4 / 5$ & 80 & 75 & 74 \\
\hline 22 & $\mathrm{~F}$ & 76 & 52 & 24 & $10 / 13 \mathrm{GCN}$ & 11 & 18 & $3 / 3$ & 5 & 0 & 64 \\
\hline 23 & $\mathrm{~F}$ & 67 & 40 & 27 & $10 / 14 \mathrm{GCN}$ & 8 & 2 & $5 / 3$ & 25 & 50 & 100 \\
\hline 24 & $\mathrm{M}$ & 62 & 48 & 14 & $10 / 13 \mathrm{GCN}$ & 0 & 5 & $0 / 3$ & 70 & 100 & 100 \\
\hline \multirow{2}{*}{\multicolumn{2}{|c|}{$\begin{array}{l}\text { Mean (SD): } \\
\text { Correlation with } \\
\text { ALS-FTD-Q }\end{array}$}} & $61.2(7.4)$ & $48.1(6.4)$ & $13.3(8.4)$ & & $14.9(13.8)$ & $11.1(7.6)$ & $3.3(2.9) / 4.0(3.5)$ & $53.3(30.4)$ & $57.6(41.6)$ & 72.9 (24.8) \\
\hline & & $0.21, p=0.334$ & $0.18, p=0.429$ & $0.12, p=0.574$ & $0.31, p=0.141$ & & & $0.22, p=0.293$ & $-0.35, p=0.104$ & $-0.39, p=0.070$ & $-0.38, p=0.075$ \\
\hline
\end{tabular}

F: female; M: male; y: years; ${ }^{\text {a }}$ : normal repeat length is 10/10; ALS-FTD-Q= Amyotrophic Lateral Sclerosis Fronto-Temporal Dementia Questionnaire; maximum score = 100; higher scores indicate more behavioural changes; *underlined and bold values indicate mild and severe behavioural changes, respectively. HADS = Hospital Anxiety and Depression Scale, values represent anxiety and depression subsets, both with maximum score of 21 ; higher scores indicate more affective symptoms. $\mathrm{PF}=\mathrm{Physical}$ Functioning; $\mathrm{RP}=\mathrm{Role} \mathrm{Physical}$; $\mathrm{BP}=\mathrm{Bodily} \mathrm{Pain} ; \mathrm{SF}=\mathrm{Socia}$ Functioning; $\mathrm{MH}=$ Mental Health; $\mathrm{RE}=$ Role Emotional; VT = Vitality; GH = General Health; DDI = Dutch Dysarthria Investigation for adults; maximum score = 28; higher scores indicate more severe dysarthria. Swallowing score; maximum score $=24$; higher scores indicate more dysphagia. 


\begin{tabular}{|c|c|c|c|c|c|c|c|}
\hline Case & $\begin{array}{c}\text { SF-36; } \\
\text { SF score }\end{array}$ & $\begin{array}{c}\text { SF-36; } \\
\text { MH score }\end{array}$ & $\begin{array}{c}\text { SF-36; } \\
\text { RE score }\end{array}$ & $\begin{array}{c}\text { SF-36; } \\
\text { VT score }\end{array}$ & $\begin{array}{c}\text { SF-36; } \\
\text { GH score }\end{array}$ & DDI score & $\begin{array}{c}\text { Swallowing } \\
\text { score }\end{array}$ \\
\hline 1 & 63 & 52 & 67 & 30 & 25 & 8 & 12 \\
\hline 2 & 100 & 88 & 100 & 85 & 77 & 0 & 0 \\
\hline 3 & 75 & 88 & 100 & 70 & 87 & 0 & 3 \\
\hline 4 & - & - & - & - & - & 6 & 1 \\
\hline 5 & 75 & 92 & 33 & 65 & 77 & 5 & 8 \\
\hline 6 & 88 & 60 & 67 & 40 & 62 & 0 & 7 \\
\hline 7 & 63 & 80 & 100 & 60 & 52 & 2 & 7 \\
\hline 8 & 75 & 88 & 100 & 45 & 77 & 10 & 10 \\
\hline 9 & 100 & 92 & 100 & 90 & 90 & 0 & 2 \\
\hline 10 & 100 & 88 & 100 & 70 & 72 & 0 & 3 \\
\hline 11 & 38 & 80 & 100 & 35 & 67 & 3 & 9 \\
\hline 12 & 100 & 84 & 100 & 75 & 90 & 0 & 6 \\
\hline 13 & 88 & 88 & 100 & 55 & 67 & 3 & 10 \\
\hline 14 & 75 & 88 & 100 & 20 & 30 & 18 & 12 \\
\hline 15 & 75 & 88 & 100 & 30 & 62 & 0 & 6 \\
\hline 16 & 50 & 80 & 100 & 55 & 45 & 6 & 8 \\
\hline 17 & 75 & 76 & 100 & 10 & 25 & 4 & 8 \\
\hline 18 & 100 & 92 & 100 & 80 & 47 & 0 & 6 \\
\hline 19 & 100 & 84 & 100 & 65 & 47 & 15 & 5 \\
\hline 20 & 63 & 64 & 33 & 45 & 67 & 12 & 8 \\
\hline 21 & 88 & 68 & 100 & 30 & 35 & 4 & 8 \\
\hline 22 & 75 & 88 & 0 & 35 & 30 & 6 & 10 \\
\hline 23 & 88 & 84 & 100 & 70 & 67 & 15 & 10 \\
\hline 24 & 100 & 92 & 100 & 80 & 67 & 0 & 2 \\
\hline Mean (SD): & 80.4 (17.6) & $81.9(11.0)$ & $87.0(28.0)$ & $53.9(22.4)$ & $59.4(20.5)$ & $4.9(5.5)$ & $6.7(3.4)$ \\
\hline $\begin{array}{l}\text { Correlation } \\
\text { with ALS-FTD-Q }\end{array}$ & $-0.36, p=0.091$ & $-0.17, p=0.449$ & $-0.07, p=0.737$ & $-0.34, p=0.113$ & $-0.36, p=0.089$ & $0.19, p=0.370$ & $0.12, p=0.585$ \\
\hline
\end{tabular}


[20]. These findings of brain involvement in OPMD should be corroborated by future pathological and imaging studies, and related to clinical data (including neuropsychological assessment).

The low correlation between the HADS scores and ALS-FTD-Q scores suggests that not all behavioural impairments detected by the ALS-FTD-Q can be related to affective symptoms, which in turn were in the severe range in $13 \%$ in our sample [6]. Thus, in addition to bvFTD symptoms, clinicians should be aware of symptoms of a depression in patients with OPMD.

Our study did not find a correlation between behavioural impairment and repeat length. However, an observation in our study worth noting is that the patient with the most GCN-repeats $(n=18$, due to a (GCN)12/(GCN)16 mutation) had the highest proxy score on the ALS-FTD-Q.

Scores of the ALS-FTD-Q have been compared between proxies and patients; while the median scores differ only slightly and not significantly, there seems to be an apparent underreporting by patients proportion wise (no severe behavioural change and only three in the mild category). In 14 cases, patients scored lower than their proxies, possibly indicating some degree of reduced disease insight, which is associated with bvFTD.

Our study has some limitations. First, the number of responders who refused participation was substantial and we were unable to examine their reasons for refusal. This high proportion may have led to selection bias, plausibly with an underestimation of the actual proportions of behavioural change in OPMD patients, since patients with more severe behavioural impairment may be unwilling or incapable of partaking in a questionnaire. The low sample size may also have resulted in insufficient power to generate strong conclusions. Second, the ALS-FTD-Q, including the cut-off scores, has not been validated in OPMD, which may have led to a bias in assessing the proportions of behavioural change. However, it has been validated in another neuromuscular disorder with bulbar and appendicular weakness (ALS patients of whom $8 \%$ had severe and $11 \%$ mild behavioural changes) and has specifically been designed in order to avoid overestimation of behavioural impairment due to motor and bulbar impairment, which are both present in OPMD [6, 21].

Future investigations, for instance longitudinal comprehensive neuropsychological examinations (including behavioural assessments or observations) in larger population-based cohorts - supported by brain imaging and post mortem pathological examinations - will further increase our knowledge of the occurrence and clinical relevance of underlying brain changes in patients with OPMD, and may form the basis for therapeutic interventions.

\section{ACKNOWLEDGMENTS}

No financial support was needed nor supplied for this research.

\section{CONFLICT OF INTEREST}

The authors have no conflict of interest to report.

\section{REFERENCES}

[1] Brais B, Bouchard JP, Xie YG, et al. Short GCG expansions in the PABP2 gene cause oculopharyngeal muscular dystrophy. Nat Genet. 1998;18(2):164-7.

[2] Blumen SC, Bouchard JP, Brais B, et al. Cognitive impairment and reduced life span of oculopharyngeal muscular dystrophy homozygotes. Neurology. 2009;73(8):596-601.

[3] Dubbioso R, Moretta P, Manganelli F, et al. Executive functions are impaired in heterozygote patients with oculopharyngeal muscular dystrophy. J Neurol. 2012;259(5):833-7.

[4] van der Sluijs BM, te Riele MGE, Hammink JKN, Ramdhani-Joosten AAJ, Snijders AH, Raz V, van Engelen BGM, Voermans NC. Oculopharyngeal muscular dystrophy with frontotemporal dementia. European Geriatric Medicine. 2017;8(1):81-3.

[5] van Engelen BGM, van Veenendaal H, van Doorn PA, van der Hoeven JH, Janssen NG, Notermans NC, et al. The Dutch neuromuscular database CRAMP (Computer Registry of All Myopathies and Polyneuropathies): Development and preliminary data. Neuromuscular Disorders. 2007;17(1):33-7.

[6] Raaphorst J, Beeldman E, Schmand B, Berkhout J, Linssen WH, van den Berg LH, et al. The ALS-FTD-Q: a new screening tool for behavioural disturbances in ALS. Neurology. 2012;79:1377-83.

[7] Zigmond AS, Snaith RP. The hospital anxiety and depression scale. Acta Psychiatr Scand. 1983;67(6):361-70.

[8] Ware JE, Jr., Sherbourne CD. The MOS 36-Item Short-Form Health Survey (SF-36): I. Conceptual Framework and Item Selection, Medical Care. 1992;30:473-83.

[9] Knuijt S, Kalf H, Gerven Van M, Kocken J, Kromhout L, Goos H, Swart Dd B. Nederlandstalig Dysartrieonderzoek Volwassenen (NDO-V), Houten, Bohn Stafleu Van Loghum. 2014.

[10] van den Engel-Hoek L, Erasmus CE, van Bruggen HW, de Swart BJ, Sie LT, Steenks MH, et al. Dysphagia in spinal muscular atrophy type II: more than a bulbar problem? Neurology. 2009;73:1787-91.

[11] Castor Electronic Data Capture, Ciwit BV, Amsterdam, The Netherlands, 2018.

[12] Cohen J. Statistical Power Analysis for the Behavioral Sciences, 2nd ed. Hillsdale, NJ: Erlbaum 1988. 
[13] Onyike CU, Diehl-Schmid J. The epidemiology of frontotemporal dementia. Int Rev Psychiatry. 2013;25(2):130-7. doi:10.3109/09540261.2013.776523

[14] Rosso SM, Donker Kaat L, Baks T, et al. Frontotemporal dementia in The Netherlands: patient characteristics and prevalence estimates from a population-based study. Brain. 2003;126(9):2016-22.

[15] Jouan L, Rocheford D, Szuto A, et al. An 18 alanine repeat in a severe form of oculopharyngeal muscular dystrophy. Can J Neurol Sci. 2014;41(4):508-11.

[16] Mizoi Y, Yamamoto T, Minami N, et al. Oculopharyngeal muscular dystrophy associated with dementia. Intern Med. 2011;50(20):2409-12.

[17] Burrell JR, Halliday GM, Kril JJ, Ittner LM, Götz J, Kiernan MC, Hodges JR. The frontotemporal dementia-motor neuron disease continuum. Lancet. 2016;388(10047):919-31.
[18] Okkersen K, Buskes M, Groenewoud J, Kessels RPC, Knoop H, van Engelen B, Raaphorst J. The cognitive profile of myotonic dystrophy type 1: A systematic review and meta-analysis. Cortex. 2017;95:143-55.

[19] Chiò A, Ilardi A, Cammarosano S, Moglia C, Montuschi A, Calvo A. Neurobehavioral dysfunction in ALS has a negative effect on outcome and use of PEG and NIV. Neurology. 2012;78(14):1085-9.

[20] Dion P, Shanmugam V, Gaspar C, et al. Transgenic expression of an expanded (GCG)13 repeat PABPN1 leads to weakness and coordination defects in mice. Neurobiol Dis. 2005;18(3):528-36.

[21] Beeldman E, Govaarts R, de Visser M, et al. Progression of cognitive and behavioural impairment in early amyotrophic lateral sclerosis [published online ahead of print, J Neurol Neurosurg Psychiatry. 2020;13:2020;jnnp-2020-322992. 